\title{
On The Use of Beryllium as Thermal Neutron Filter
}

\author{
M. Wahba \\ Faculty of Engineering, Ain Shams University Cairo, Egypt
}

A formula is given which allows to calculate the contribution of the nuclear capture, thermal diffuse and Bragg scattering cross-section to the neutron transmission through solid crystalline Beryllium. The formula takes into account the beryllium crystalline structure and its physical parameters. A computer program CRYSBE was developed to provide the required calculation. The calculated values of the total cross-section of polycrystalline beryllium at different temperatures were compared with the previously measured ones in the neutron energy range from $0.2 \mathrm{meV}$ to $10 \mathrm{eV}$. The obtained agreement shows that the formula fits the experimental data. An agreement was also obtained for values of beryllium single crystal at both room and liquid nitrogen temperatures. The feasibility study on using a polycrystalline beryllium as a cold neutron filter is given. The optimum beryllium single crystal thickness, mosaic spread, temperature and cutting plane for efficiently transmitting the thermal reactor neutrons, while rejecting both fast neutrons and gamma ray accompanying the thermal ones are also given . 


\section{Introduction:}

A fission reactor is a prolific source of fast neutrons, thermal neutrons and gamma radiation. However, to improve the signal-to-background ratio for thermal neutron scattering experiments has required the development of thermal neutron filters .

For most media the reflective index for neutrons is less than 1.0. It follows that when a neutron beam strikes the boundary of a medium from outside (i.e. from vacuum), one should observe an almost total internal reflection of neutrons from the surface at small enough glancing angles .As shown by several authors $[1,5]$, curved guide tubes transports neutrons by total internal reflection from a surface coating of ${ }^{58} \mathrm{Ni}$ mirror .

Such thermal neutron filters are expensive to construct and the neutron scattering facility are usually installed far away from the reactor core .

However, $\mathrm{Si}, \mathrm{Be}, \mathrm{Cu}$ and graphite poly and momo-crystals have been recently used as thermal neutron filters[6,7]. When evaluating crystal filters that pass thermal neutrons and exclude both fast neutrons and $\gamma$-rays, the material used must be of small absorption cross-section and high free atom scattering cross section for thermal neutron. Therefore the present work deals with the feasibility study on using a poly and mono-crystalline beryllium as a thermal neutron filter, since its absorption cross section is less than 0.002 barns while its free atom cross section is 7.6barns.

A simple formula was introduced for calculating both the total thermal cross-section and Bragg scattering cross-section of a beryllium in poly and mono crystalline form.

The computer program CRYSBE, a new version of computer code ISCANF- II[8] was adapted to provide the required calculation.

\section{The Theoretical Treatment}

\section{Attenuation of neutrons by a crystalline solid :}

The total cross section determining the attenuation of neutrons by crystalline solid is given by

$$
\sigma=\sigma_{\mathrm{abs}}+\sigma_{\mathrm{tds}}+\sigma_{\mathrm{Bragg}}
$$

The first contribution $\sigma_{\text {abs }}$ for the most of the elements obeys the $1 / v$ law, where $\mathrm{v}$ is the neutron velocity and can be written as: 


$$
\sigma_{\mathrm{abs}}=\mathrm{C}_{1} \mathrm{E}^{-1 / 2}
$$

where $\mathrm{E}$ is the energy of the incident neutron

According to Freund [9] the second contribution $\sigma_{\mathrm{tds}}$ can be split into two parts, $\sigma_{\mathrm{sph}}$ and $\sigma_{\mathrm{mph}}$ depending on neutron energy. The single-phononscattering cross section $\sigma_{\text {sph }}$ concerns the energy range $E \ll K_{B} \theta_{D}$, where $K_{B}$ is Boltzmann' s constant and $\theta_{\mathrm{D}}$ is the characteristic Debye temperature that is given by the equation:

$$
\sigma_{\text {sph }}=\frac{\sigma_{\text {bat }}}{36 \mathrm{~A}}\left(\frac{\theta_{\mathrm{D}}}{\mathrm{E}}\right)^{1 / 2}\left\{\begin{array}{cc}
\mathrm{R} & \mathrm{x} \leq 6 \\
3.3 \mathrm{x}^{-7 / 2} & \mathrm{x} \geq 6
\end{array}\right.
$$

where $x=\theta_{\mathrm{D}} / \mathrm{T}$ ( $\mathrm{T}$ is the temperature $), \sigma_{\mathrm{bat}}=\mathrm{S}+\mathrm{s}$ the sum of coherent and incoherent scattering cross section of the bound atom, $\mathrm{A}$ is the mass number and

$$
\mathrm{R}=\sum_{\mathrm{n}=0}^{\infty} \mathrm{B}_{\mathrm{n}} \mathrm{x}^{\mathrm{n}-1} /[\mathrm{n} !(\mathrm{n}+5 / 2)]
$$

where $B_{n}$ are the Bernoulli numbers.

The second part, the multi-phonon scattering $\sigma_{\mathrm{mph}}$ of the $\sigma_{\mathrm{tds}}$ is predominant in the range $E \gg K_{B} \theta_{D}$ and is given by:

$$
\sigma_{\text {mph }}=\sigma_{\text {free }}\left\{1-\exp \left[-\left(B_{o}+B_{T}\right) C_{2} E\right]\right\}
$$

where $\mathrm{C}_{2}$ is a constant independent of the scattering material,

$$
\begin{aligned}
& \mathrm{B}_{\mathrm{o}}=3 \mathrm{~h}^{2} /\left(2 \mathrm{~K}_{\mathrm{B}} \theta_{\mathrm{D}}\right) \\
& \mathrm{B}_{\mathrm{T}}=4 \mathrm{~B}_{\mathrm{o}} \varphi(\mathrm{x}) / \mathrm{x}
\end{aligned}
$$

in which $\mathrm{h}$ is plank's constant and

$$
\varphi(\mathrm{x})=\mathrm{x}^{-1} \int_{0}^{\mathrm{x}} \zeta \mathrm{d} \zeta /\left(\mathrm{e}^{-\zeta}-1\right)
$$

$\sigma_{\text {free }}$ is the free atom cross section is given by

$$
\sigma_{\text {free }}=\sigma_{\text {bat }}[\mathrm{A} / \mathrm{A}+1]^{2}
$$

\section{Bragg Scattering :}

The contribution of Bragg scattering $\sigma_{\text {Bragg }}$ to the total cross section taking into account the resulting reflection from different $(h k l)$ planes, which are able of giving the Bragg reflection for the neutron wavelength $\lambda$, was 
calculated. In case of a poly- crystalline material the reflections are from all planes having spacing $\mathrm{d}_{h k l} \geq \lambda / 2$, while in case of a mono crystal, reflections are from the $(h k l)$ planes satisfying the Bragg equation:

$$
\mathrm{n} \lambda=2 \mathrm{~d}_{h k l} \sin \theta_{h k l}
$$

where $\mathrm{n}$ is the order of reflection, $\theta_{h k l}$ is the glancing angle to the $(h k l)$ plane.

\section{1. Bragg Scattering by a Polycrystalline Material:}

It was shown by Bacon [10] that for a Polycrystalline material with grain size less than $10^{-4} \mathrm{~mm}$, the total coherent Bragg scattering cross-section can be given as:

$$
\sigma_{\text {Bragg }}=\frac{N_{c} \lambda^{2}}{2} \sum_{d_{\mathbf{h k l}} \geq \lambda / 2} F_{\mathbf{h k l}}^{2} d_{\mathbf{h k l}} \cdot e^{-2 w}
$$

where $\mathrm{N}_{c}$ is the number of unit cells per cubic centimeter, $\mathrm{F}_{h k l}$ is the structure factor of the unit cell and $\mathrm{e}^{-2 \mathrm{w}}$ is the Debye-Waller factor.

\section{2. Bragg Scattering by a Single Crystal:}

Following Naguib K. and Adib M., [11], the Bragg scattering crosssection by a single crystal is given by:

$$
\sigma_{\text {Bragg }}=\frac{1}{N t_{o}} \ln \left(\frac{1}{T_{\text {Bragg }}}\right)
$$

where $\mathrm{N}$ is the number of atoms per cubic centimeter and $\mathrm{t}_{\mathrm{o}}$ is the effective thickness of the crystal in $\mathrm{cm}$. $\mathrm{T}_{\text {Bragg }}$ is the resulting neutron transmission from different $(h k l)$ planes given by:

$$
\mathrm{T}_{\mathrm{Bragg}}=\prod_{h k l}\left(1-P_{h k \mathrm{l}}^{\theta}\right)
$$

where $P_{h k l}^{\theta}$ is the reflecting power of the $(h k l)$ plane inclined by an angle $\theta_{h k l}$ to the incident beam direction.

As shown the authors [11] the reflecting power $P_{h k 1}^{\theta}$ for an ideally imperfect crystal depends upon the direction cosine of the incident beam $\gamma_{0}$ relative to the inward normal to the crystal surface cutting along the plane 
$\left(h_{c} k_{c} l_{c}\right)$, the direction cosine of the diffracted beam $\gamma_{h k l}$ and the inclination of ( $h k l)$ plane to the crystal surface $\alpha_{h k l}$.

For the hexagonal closed pack structure(hcp), the equation describing a cutting plane with miller indices $\left(h_{c} k_{c} l_{c}\right)$ can be given as:

$$
\frac{h_{c}}{a_{o}} X+\frac{2}{\sqrt{3} a_{o}}\left(\frac{h_{c}}{2}+k_{c}\right) Y+\frac{l_{c}}{c_{o}} Z=1
$$

when such crystal is fixed on a goniometer's table with its surface is parallel to $\mathrm{XY}$ plane, the equation of its cutting plane using a simple plane transformation can be given as :

$$
\mathrm{Z} / \mathrm{d}_{\mathrm{C}}=1
$$

while any of the $(h k l)$ planes can be given as:

$$
\begin{aligned}
& =\frac{\mathrm{M}_{\mathrm{o}}}{\mathrm{a}_{\mathrm{o}}}\left(\mathrm{hk}_{\mathrm{c}}-\mathrm{kh}_{\mathrm{c}}\right)+\mathrm{d}_{\mathrm{c}} \frac{21}{\sqrt{3 a_{O} c_{o}}}\left[M_{c}\left(h h_{c}+k k_{c}\left(k h_{c}+h k_{c}\right) / 2-\frac{1}{M c}\right)\right] Y \\
& +\mathrm{dc}\left[\frac{4}{3 \mathrm{a}_{\mathrm{o}}^{2}}\left\{h h_{c}+k k_{c}+\left(h k_{c}+k h_{c}\right) / 2\right\}+l l c / c_{O}^{2}\right] \mathrm{Z}=1
\end{aligned}
$$

where

and,

$$
M_{c}=\frac{1}{\sqrt{h_{c}^{2}+k_{c}^{2}+h_{c} k_{c}}}
$$

$$
d_{c}=\frac{1}{\sqrt{\frac{4}{3 a_{o}^{2}}\left(h_{c}^{2}+k_{c}^{2}+h_{c} k_{c}\right)+l_{c}^{2} / c_{o}^{2}}}
$$

where $\mathrm{a}_{\mathrm{o}}, \mathrm{c}_{\mathrm{o}}$ are a lattice constant.

If the angle between the neutron beam direction and the direction $\left[h_{c} k_{c} l_{c}\right]$ is $\psi$, then the direction cosine of the diffracted beam $\gamma_{h k l}$ can be expressed as: 


$$
\begin{aligned}
& \gamma_{\mathrm{hkl}}=\mathrm{d}_{\mathrm{c}} \mathrm{d}_{\mathrm{hkl}}\left[\frac{4}{3 \mathrm{a}_{\mathrm{O}}^{2}}\left\{h h_{c}+k k_{c}+\left(h k_{c}+k h_{c}\right) / 2\right\}+l l c / c_{O}^{2}\right] \cos \psi+ \\
& \frac{2 l}{\sqrt{3 a_{O} c_{O}}}\left[M_{c}\left(h h_{c}+k k_{c}\left(k h_{c}+h k_{c}\right) / 2-\frac{1}{M c}\right)\right] \sin \psi
\end{aligned}
$$

where

$$
d_{h k l}=\frac{1}{\sqrt{\frac{4}{3 a_{o}^{2}}\left(h^{2}+k^{2}+h k\right)+l^{2} / c_{o}^{2}}}
$$

while the inclination angle $\alpha_{h k l}$ of any plane $(h k l)$ to the cutting plane $\left(h_{c} k_{c} l_{c}\right)$ can be given as:

$$
\cos \alpha_{\mathrm{hkl}}=\mathrm{d}_{\mathrm{hkl}} \mathrm{d}_{\mathrm{c}}\left[\frac{h h_{c}}{\mathrm{a}_{\mathrm{o}}^{2}}+\frac{4}{3 \mathrm{a}_{\mathrm{o}}^{2}}\left(\frac{h}{2}+k\right)\left(\frac{h_{c}}{2}+k_{c}\right)+\frac{l l c}{c_{\mathrm{o}}^{2}}\right]
$$

If the cutting plane is $\left(00 l_{c}\right)$ equations (7) will be:

and

$$
\gamma_{\mathrm{hkl}}=\mathrm{d}_{\mathrm{hkl}}\left[\frac{l}{\mathrm{c}_{\mathrm{o}}} \cos \psi+\left\{\frac{2}{\sqrt{3} \mathrm{a}_{\mathrm{o}}}\left(\frac{\mathrm{h}}{2}+k\right)\right\} \sin \psi\right] \text { (9) }
$$

$$
\cos \alpha_{h k l}=\mathrm{d}_{h k l} \frac{l}{\mathrm{c}_{\mathrm{O}}}
$$

\section{Description of Crysbe Code}

CRYSBE code is an adapted version of ISCANF-I and ISCANF-II codes developed to calculate the total neutron cross-section and transmission through crystalline materials for neutron energies below $10 \mathrm{eV}$ [12]. The contribution of $\sigma_{\mathrm{abs}}$, and $\sigma_{\mathrm{tds}}$ are calculated in a similar way as given in ISCANF-I and ISCANF-II programs. The adapted version CRYSBE can provide additionally the following calculations:

1- The Bragg scattering term for a poly-and mono -crystal for HCP having two atoms per unit cell.

2- The energy and wavelength distribution of incident reactor neutron flux before and after its transmission through the crystalline Be. 


\section{Comparison with Experiment:}

In order to check the applicability of the deduced formula, the calculations were carried out for poly and mono crystalline beryllium crystals and compared with the experimental ones. The main beryllium physical parameters used for calculations are listed in Table (1).

Table .(1) The physical parameters of beryllium

\begin{tabular}{|ll|}
\hline Atomic Weight & 9.0 \\
Crystal Structure & $\mathrm{hcp}$ \\
Lattice Constants & $\mathrm{a}_{0}=0.2275 \mathrm{~nm} \mathrm{C}_{\mathrm{o}}=0.358 \mathrm{~nm}$ \\
Atomic Positions & $1 / 32 / 3 \quad 1 / 4,2 / 3 \quad 1 / 3 \quad 3 / 4$ \\
Number of unit cells $/ \mathrm{m}^{3}$ & $0.6165 \mathrm{E}+29$ \\
Debye Temperature & $1100 \mathrm{~K}$ \\
Neutron capture cross-section at $0.025 \mathrm{eV}$ & $1.68 \mathrm{mbarn}$ \\
$\sigma_{\text {bat }}$ & $7.631 \mathrm{barns}$ \\
Coherent Scattering amplitude & $7.746 \mathrm{fm}$ \\
\hline
\end{tabular}

\section{Polycrystalline Beryllium}

The total neutron cross-sections of beryllium at $400 \mathrm{~K}$, room and liquid nitrogen temperatures were calculated in the energy range from $0.1 \mathrm{meV}$ up to $10 \mathrm{eV}$ using CRYSBE. The result of calculations are displayed in Fig. (1) as solid lines. For comparison the available experimental values measured for polycrystalline beryllium in powder form at $400 \mathrm{k}$, room(R.T) and liquid(L.N) nitrogen temperatures [9], are also displayed in Fig. (1) as dots. The calculated values at $400 \mathrm{~K}$, and room temperatures are in reasonable agreement with the experimental ones at the fitted parameter $\mathrm{C}_{2}=7.6$. This value is more than that value deduced from the semi empirical formula $\left(\mathrm{C}_{2}=5.0 \AA^{-2} \mathrm{eV}^{-1}\right)$ reported by Freund [9]. However at liquid Nitrogen temperatures the calculated values are in slight disagreement with measured ones. Such disagreement is due the fact that single phonon scattering cross-section is calculated using an approximate equation at $x=13$. More exact calculation would need a detailed knowledge of the phonon frequency distribution

To show the effect of both thickness and temperature of the polycrystalline beryllium on its filtering characteristics, the calculations were performed at room and liquid nitrogen temperatures in the neutron wavelength from $0.2 \mathrm{~nm}$ up to $1.0 \mathrm{~nm}$. The result of calculation is displayed in Fig.(2). At thickness of $20 \mathrm{~cm}$ the neutron transmission at wavelength longer than the cut off wavelength at $0.396 \mathrm{~nm}$ is about $80 \%$.

Fig.(3) displays the calculated thermal neutron flux having Maxwellian distribution with neutron gas temperature close to room temperatures $(300 \mathrm{~K})$ 
incident on a $20 \mathrm{~cm}$ thick polycrystalline beryllium cooled at liquid nitrogen and after its transmission. Fig.(3) shows that polycrystalline beryllium cooled at liquid nitrogen temperature can be efficiently used as a cold neutron filter. Moreover, at such thickness the attenuation factor of $\gamma$-rays accompanying the reactor neutrons with average energy $\mathrm{E} \gamma \approx 2 \mathrm{MeV}$ is more than 50 .

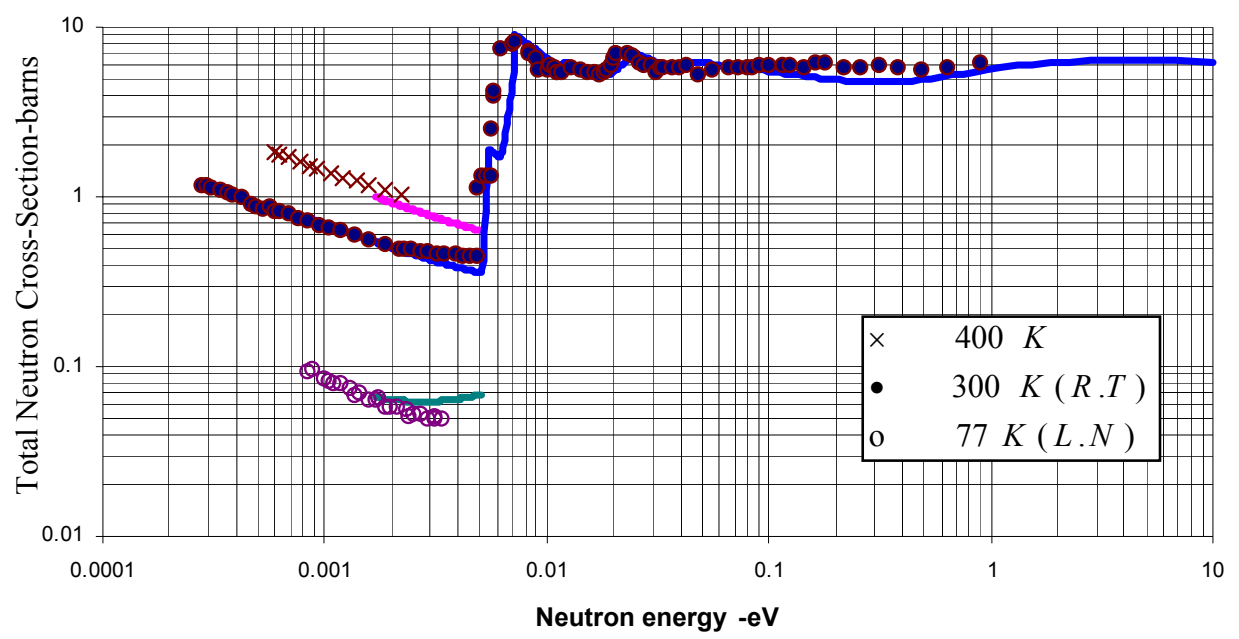

Fig .(1): The Total Neutron Cross-Section For Polycrystalline Beryllium .

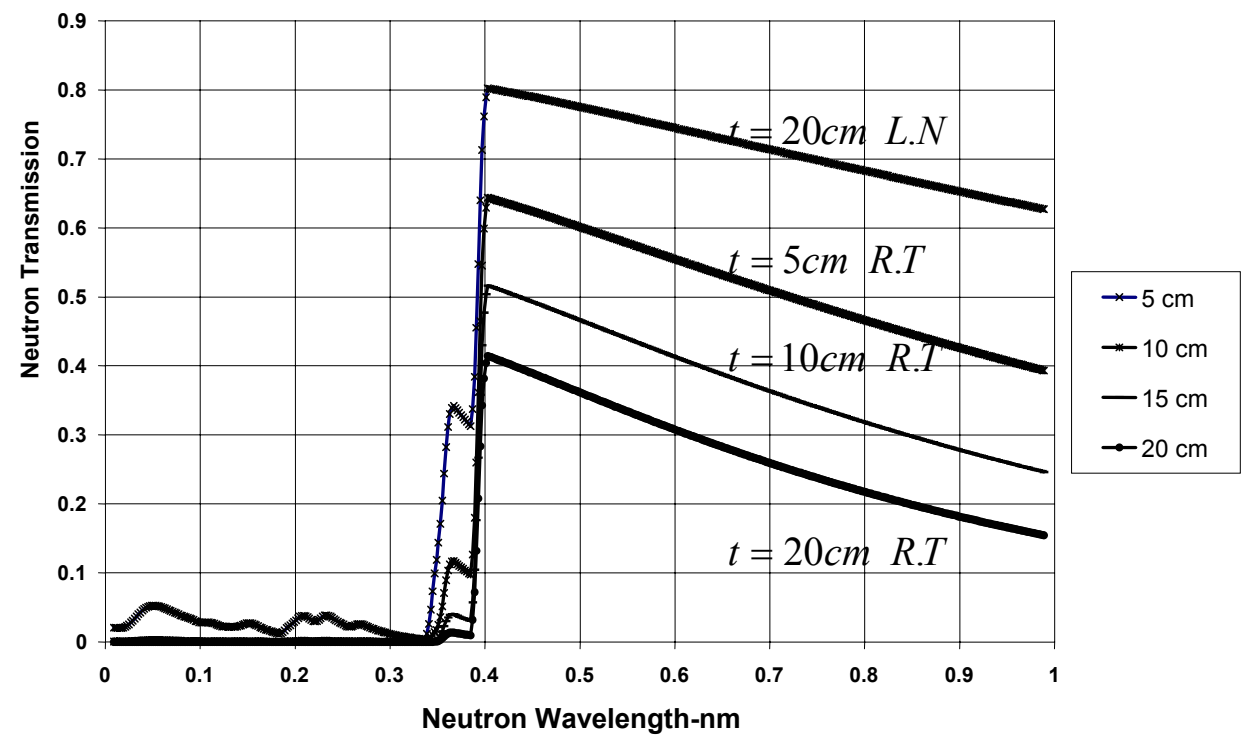

Fig.(2): Neutron Transmission through Polycrystalline Beryllium. 


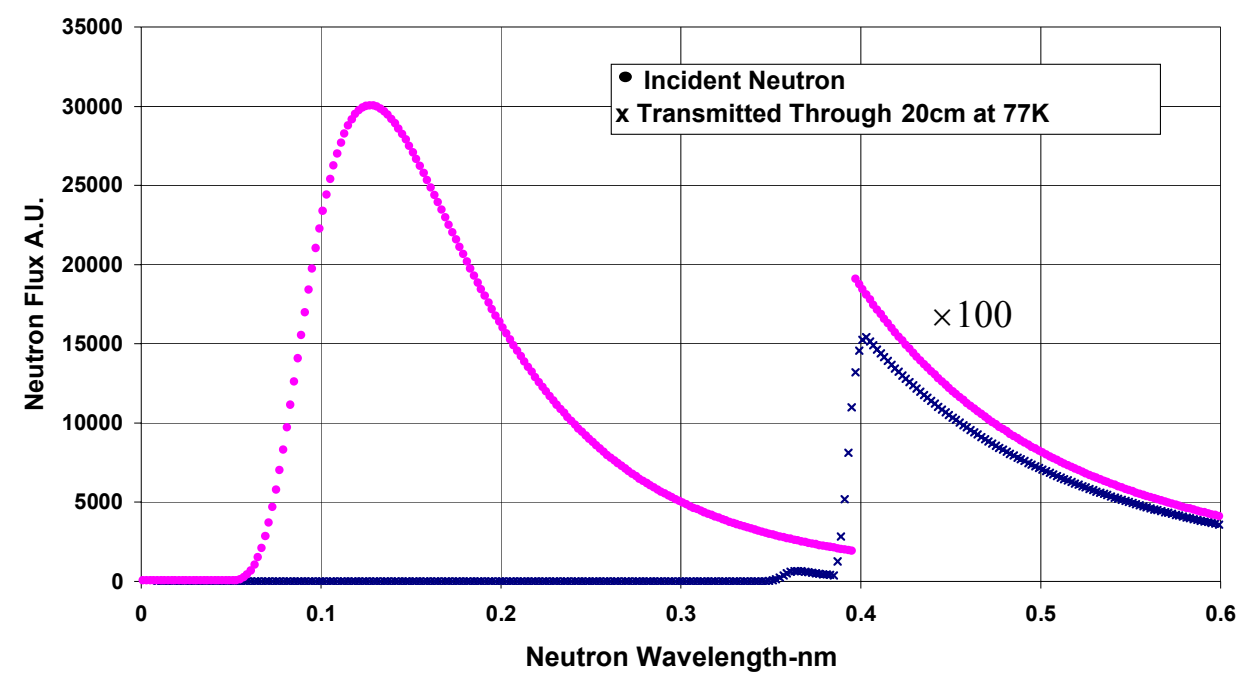

Fig.(3): Transmitted Thermal Neutron Flux through Polycrystalline Beryllium .

\section{2- Beryllium single crystal:}

The measured neutron cross-section data of beryllium single crystal reported by Freund et al [9] at room and liquid nitrogen temperatures are displayed in Fig.4. The neutron cross-section values in the energy range from $0.5 \mathrm{meV}$ to $10 \mathrm{eV}$ at room and liquid nitrogen temperatures were calculated using CRYSBE code assuming that beryllium is a perfect single crystal cut along its (002) plane. The result of calculation is displayed in Fig. (4) as solid lines. From Fig.(4) the calculated and experimental cross-section are in reasonable agreement at room temperature, however at liquid nitrogen one a disagreement was also observed as for a polycrystalline case.

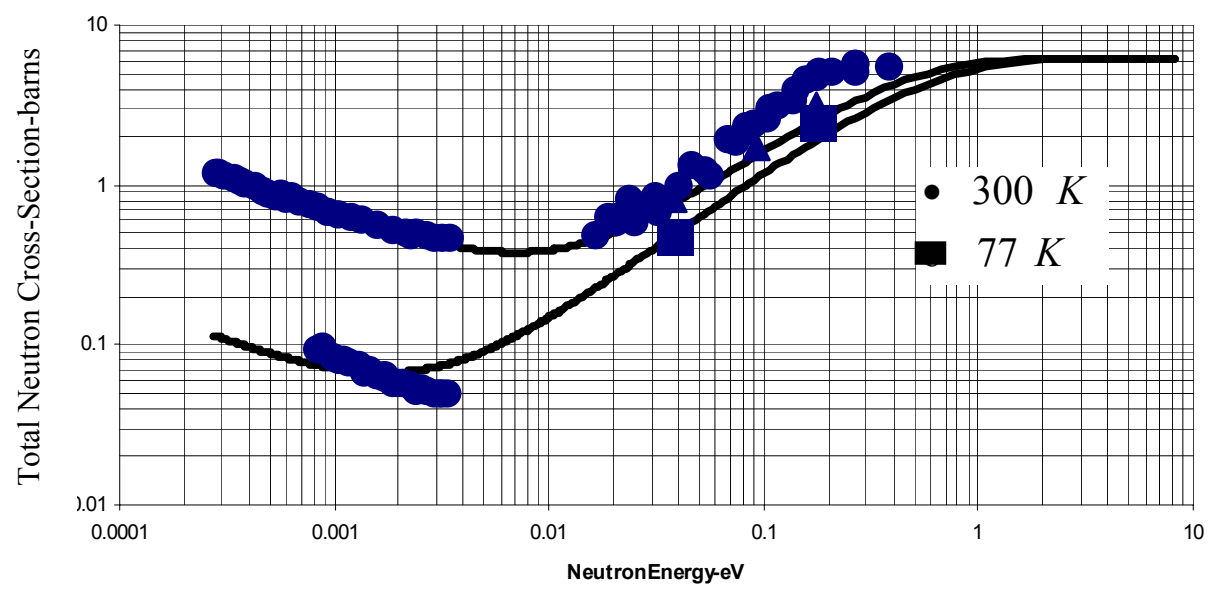

Fig .(4): Neutron Cross-Section of a Perfect Beryllium Single Crystal . 
To show the contribution of the Bragg reflections on the neutron transmission of beryllium single crystal cut along different $\left(h_{c} k_{c} l_{c}\right)$ planes, the calculations were carried out using CRYSBF code in the whole energy range from $1 \mathrm{meV}$ to $10 \mathrm{eV}$. The beryllium single crystal has a same thickness of $10 \mathrm{~cm}$ mosaic spread of $8 \mathrm{mRad}$ and at room temperature. Fig.(5) displays the result of calculations of neutron transmission through beryllium single crystals cut along (002) , (100), (101) and (103) planes for neutrons incident in a direction perpendicular to the cutting plane i.e $\psi=0$.
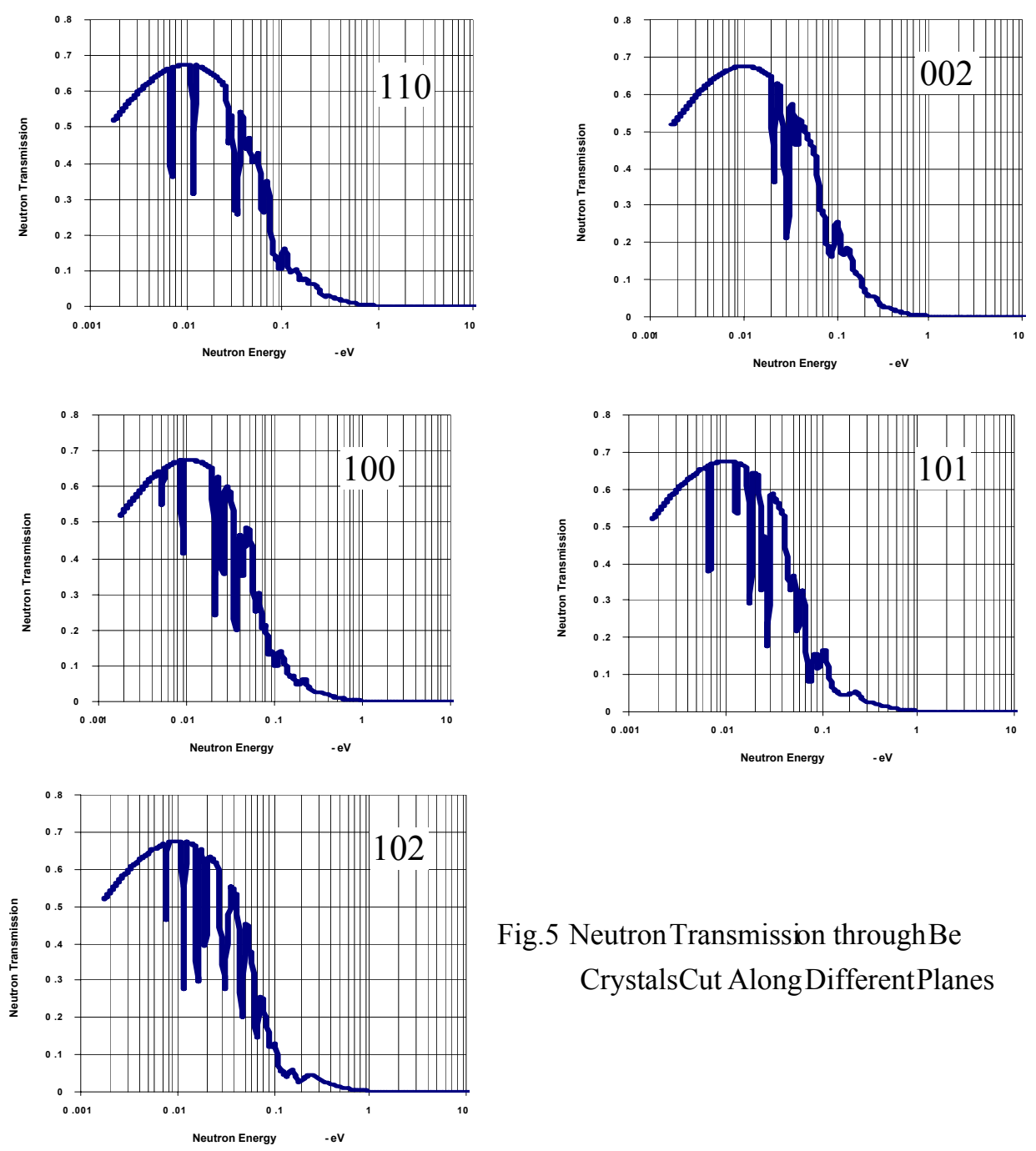

Fig.5 NeutronTransmission throughBe CrystalsCut Along DifferentPlanes 
From the curves one can notice that beryllium crystal cut along the (002) plane is preferable than the others, when it is used as a thermal neutron filter, since there are no disturbing Bragg reflection at neutron energies less than $0.02 \mathrm{eV}$. To decrease such effect a optimum choice of crystal mosaic spread is essential . The neutron transmission through $10 \mathrm{~cm}$ beryllium single crystals $(002)$ cooled at liquid nitrogen having different mosaic spread were calculated Fig. (6) displays the results of calculations. One can notice that for mosaic spread more than $1 \mathrm{mRad}$ the parasitic Bragg reflections could limits the use of beryllium single crystal as a thermal neutron filter .
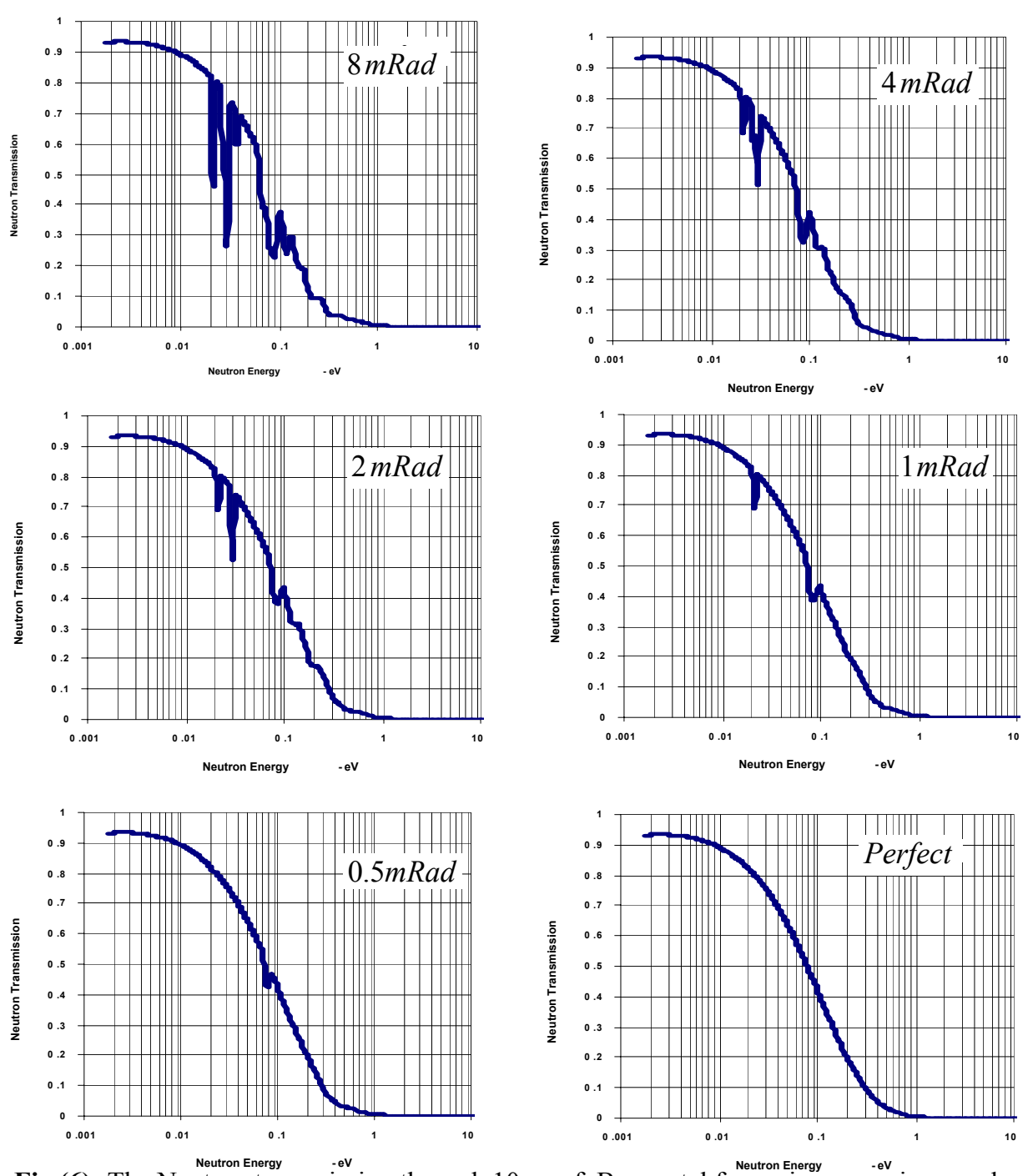

Fig.(6): The Neutron transmission through $10 \mathrm{~cm}$ of Be crystal for various mosaic spreads. 
To find the optimum beryllium thickness when used as a filter, the neutron transmission through different crystal thicknesses cooled at liquid nitrogen were calculated. Fig.7a shows the result of calculation for neutron transmission through beryllium (002) crystal having mosaic spread of $0.5 \mathrm{mRad}$ while Fig.(7b) for $1 \mathrm{mRad}$.

It seems that $10 \mathrm{~cm}$ thick $0.5 \mathrm{mRad}$ beryllium (002) cooled at liquid nitrogen is sufficient for removing neutrons with energies $>0.5 \mathrm{eV}(\mathrm{T}<3 \%)$ while providing high transmission $(\mathrm{T}>80 \%)$ for neutron energies $<0.025 \mathrm{eV}$. The transmitted neutron spectrum is almost free from disturbing Bragg reflections. The filtering characteristics are somehow worth for crystal having mosaic spread of $1 \mathrm{mRad}$. The final choice depends upon the experimental conditions required and the price of such crystal .
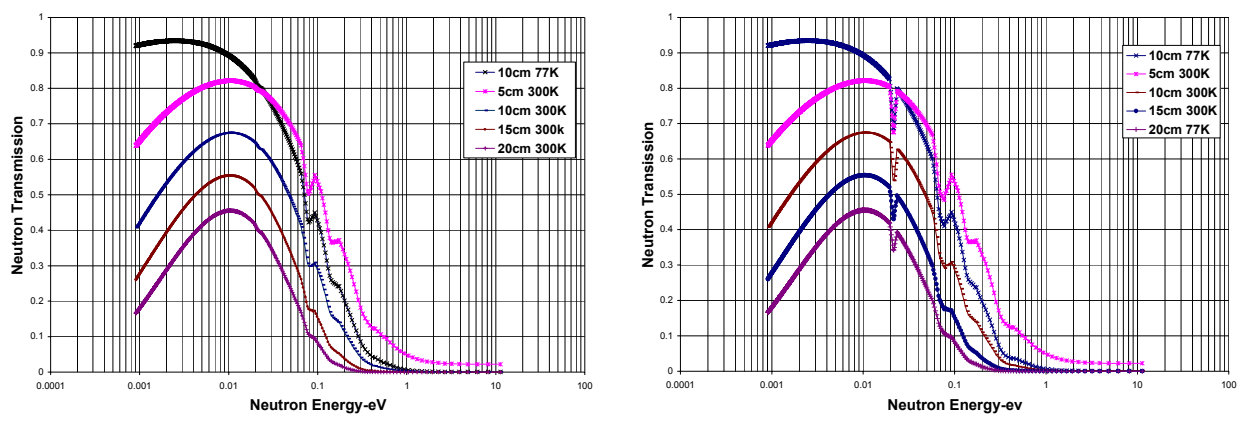

Fig.(7): Neutron Transmission through Beryllium Crystals Cut along (002) Plane for D0ifferent Crystal Thickness

\section{Conclusion:}

The simple formula and the developed CRYSBE code presented in this paper permits the calculation of the total neutron cross-section of poly- and mono-beryllium crystals with HCP structure to be deduced within an accuracy which is sufficient for determining its filtering characteristics .

Calculation shows that $20 \mathrm{~cm}$ thick polycrystalline beryllium cooled at liquid nitrogen temperature can be efficiently used as a cold neutron filter. While $10 \mathrm{~cm}$ thick beryllium single crystal cut along its (002) plane and with mosaic spread of $0.5 \mathrm{mRad}$ is a good thermal neutron one with high noise-tobackground ratio. 


\section{References:}

1. D.A.Korneev.; V.V.Pasyuk, and, A.V.Petrenko. Proceedings of the $26^{\text {th }}$. Zakopane School on Physics. Zakopane, Poland I3-2. Singapore. World Scientific Publishing Company. 401 p. p. 326-335 April (1991).

2. J.Y.Kim,.;B.W.Wehring,;K.Uenlue,Transactions-of-the-AmericanNuclear Society., 68, 162 (1993).

3. A.E.Munter, B.J.Heuser, K.M.Skulina, Physica.- B.Condensed Matter. 221(1-4), 500 (1996).

4. K.Ibel, Guide to Neutron Research Facilitates at ILL, Grenoble (1994).

5. A. Funer, New Instruments and Science Around SINQ, PSI Villigen, Switzerland, Aug.(1996)

6. R. M.Brugger., R. G.Fluharty, P. W.Lisowski and C. E.Olsen; Int. Conf. On Cross-Sections for Technology, Knoxville, Tennessee (1979).

7. J. A.Harvey, H. A.Mook, N. W.Hill and O.Shahal; Int. Conf. on Nuclear Data for Science and Technology, Antwerp (1982).

8. M. Adib, K. Nagiub, A. Ashry and M. Fathallah, Ann. Nucl. Energy, 29, 1119 (2001).

9. A.K. Freund, Nucl. Instr \& Meth., 213, 495 (1983).

10. G. E.Bacon; Neutron Diffraction $3^{\text {rd }}$ Edn. Oxford Claredon (1973).

11. K.Nagiub and M.Adib, J. Appl.Phys D., 29, 1441 (1996).

12. K.Nagiub and M.Adib Ann. Nucl. Energy., 25, No.,(18), 1553 (1998). 